\title{
Syntheses and Dielectric Properties of the 9-Acridinealdehyde Acetal of Poly(vinyl alcohol) and the 3-Pyrenealdehyde Acetal of Poly(vinyl alcohol)
}

\author{
Yasuo Oshiro, Masaaki Yokoyama, Yasuhiko Shirota, \\ and Hiroshi Mikawa \\ Department of Applied Chemistry, Faculty of Engineering, \\ Osaka University, Suita, Osaka, Japan. \\ (Received May 1, 1973)
}

\begin{abstract}
The 9-acridinealdehyde acetal of poly(vinyl alcohol) [abbreviated as acetal (I)] and the 3-pyrenealdehyde acetal of poly(vinyl alcohol) [abbreviated as acetal (II)] were synthesized from poly(vinyl alcohol) and 9-acridinealdehyde or 3-pyrenealdehyde, with hydrochloric acid as a catalyst, in dioxane-water binary solvents at an elevated temperature.

The degree of acetalization did not exceed $34.2 \%$ and $54.15 \%$ for acetals (I) and (II), respectively. The statistical calculation of the maximum degree of acetalization with polyacenealdehydes was made by extending the method of Flory for the formalization of poly(vinyl alcohol); here the steric hindrance caused by the bulky polyacene ring was also taken into account. The results of the calculation agreed fairly well with the experimental results.

The permittivities of the films of the acetylated acetals (I) and (II) and their chargetransfer complexes with tetracyanoquinodimethane(TCNQ) were measured at room temperature. It was found that the permittivities of both of the acetylated acetals (I) and (II) increased by complexation with TCNQ.

KEY WORDS 9-Acridinealdehyde / 3-Pyrenealdehyde / Poly(vinyl alcohol) / Acetal / Maximum Degree of Acetalization / permittivities / Charge-Transfer Complex /
\end{abstract}

Polymers having rather high permittivities have been reported by many authors. They are mostly polar polymers having polar side groups, the permittivities of which are mainly due to dipole orientation. Recently, Saito, et al., ${ }^{1}$ reported that the permittivity of the $p$-aminostyrene-methylacrylate copolymer increased when complexed with chloranil. On the other hand, we could not find any increase in the permittivities of such polymers as the 9-anthraldehyde acetal of poly(vinyl alcohol) or poly- $N$ vinylcarbazole by complexation with tetracyanoquinodimethane(TCNQ). ${ }^{2}$ In the present study new polymers such as the 9-acridinealdehyde acetal of poly(vinyl alcohol) (I) and the 3pyrenealdehyde acetal of poly(vinyl alcohol) (II) were synthesized, and their permittivities and charge-transfer complexes with TCNQ were examined. Also, a calculation of the maximum degree of acetalization of poly(vinyl alcohol) with polyacenealdehyde was made by taking account of the steric hindrance caused by the bulky polyacene ring, and was compared with the experimental results.

\section{EXPERIMENTAL}

\section{Materials}

Commercially available poly(vinyl alcohol) (Kurare poval 105, saponification degree 99\%, average polymerization degree 500) was washed with methanol several times and dried under reduced pressure. 9-Acridinealdehyde was synthesized as described in the literature $e^{4}$ and recrystallized from methanol, $\mathrm{mp} 146-147^{\circ} \mathrm{C}$. 3Pyrenealdehyde was synthesized from pyrene by means of the Vilsmeier's reaction and recrystallized from benzene- $n$-hexane, $\operatorname{mp} 126-127^{\circ} \mathrm{C} .^{5}$ Commercially available tetracyanoquinodimethane(TCNQ) was recrystallized from aceto- 
nitrile and sublimed. Dioxane, acetic anhydride, and other reagents were purified as usual.

Synthesis of the 9-Acridinealdehyde Acetal of Poly(vinyl alcohol)

PVA $(2.2 \mathrm{~g})$ and the hydrochloric acid salt of 9-acridinealdehyde $(4.0 \mathrm{~g})$ were dissolved in 100 $\mathrm{m} l$ of dioxane-water $(60: 40)$, and $8 \mathrm{~m} l$ of hydrochloric acid $(12 N)$ was added with stirring at $80^{\circ} \mathrm{C}$. The yellow solution turned to red. After $48 \mathrm{hr}$ the solution was poured into a large amount of water, and neutralized with an aqueous solution of sodium acetate. The precipitated polymer was filtered, washed first with water, then with methanol, and dried under reduced pressure to give a pale green solid with melting range $234-251{ }^{\circ} \mathrm{C}$.

Synthesis of the 3-Pyrenealdehyde Acetal of Poly(vinyl alcohol)

PVA $(1.1 \mathrm{~g})$ and 3-pyrenealdehyde $(3.0 \mathrm{~g})$ were dissolved in $100 \mathrm{~m} l$ of dioxane-water $(80: 20)$, and $8 \mathrm{~m} l$ of hydrochloric acid $(12 N)$ was added with stirring at $70^{\circ} \mathrm{C}$. After $24 \mathrm{hr}$ the solution was poured into a large amount of water and was treated in a manner similar to that described above. The 3-pyrenealdehyde acetal of poly(vinyl alcohol) thus obtained was a white fibrous mass with melting range $190-212^{\circ} \mathrm{C}$.

Acetylations of the Acetals

Since the acetals (I) and (II) were insoluble in ordinary organic solvents, acetylations of these acetals were carried out using the method of W. O. Herrman. ${ }^{6}$

The acetal $(0.5 \mathrm{~g})$ and sodium acetate $(2.5 \mathrm{~g})$ were refluxed in acetic anhydride $(50 \mathrm{ml})$ for $4 \mathrm{hr}$. The reaction solution was poured into a large excess of water and the precipitated polymer was filtered, washed with water, reprecipitated from acetone-water, dried, and reprecipitated further from methylene chloride- $n$ hexane and dried under reduced pressure.

The acetylated 9-acridinealdehyde acetal of poly(vinyl alcohol) thus obtained was a pale yellow powder with melting range $134-144^{\circ} \mathrm{C}$. It was easily soluble in acetone, methanol, and methylene chloride, but insoluble in benzene or $n$-hexane. Elementary analysis-Found: C, 65.72; H, 6.10; N, 2.55. Calcd for A.D. 34.2\%: C, 65.91; H, 6.11; N, 2.51\%. IR (solid film, $\left.\mathrm{cm}^{-1}\right): \quad \nu_{\mathrm{CH}} 3030,2910$, and $2850 ; \quad \nu_{\mathrm{C}=\mathrm{O}} 1720$;
$\delta_{\mathrm{CH}} 1420$ and $1360 ; \nu_{\mathrm{C}-\mathrm{O}-\mathrm{C}} 1240 ; \nu_{\mathrm{O}-\mathrm{C}-\mathrm{O}} 1120$ and $1020 ; \delta_{\mathrm{C}-\mathrm{O}} 940 ; \delta_{\mathrm{CH}} 710$ (acridine ring). NMR (hexadeuterio acetone): $\tau 8.60$ (multiplet, $\mathrm{CH}_{2}$ in acetal ring); $\tau 8.20$ (multiplet, $\mathrm{CH}_{2}$ ); $\tau 7.95$ (singlet, $-\mathrm{O}-\mathrm{CO}-\mathrm{CH}_{3}$ ); $\tau 6.30 \quad$ (multiplet, $\mathrm{O}>\mathrm{CH}$-acridine); $\tau 5.10$ (multiplet, $-\mathrm{CH}-\mathrm{O}-$ $\mathrm{CO}-$ ); $\tau 1.2-2.3$ (multiplet, acridine ring protons).

The acetylated 3-pyrenealdehyde acetal of poly(vinyl alcohol) was a white powder with melting range $112-124^{\circ} \mathrm{C}$, and was easily soluble in acetone, methylene chloride, and tetrahydrofuran, but insoluble in $n$-hexane. Elementary analysis-Found: C, 64.53; H, 6.29. Calcd for A.D. 20.4\%: C, 64.52; H, 6.46\%. IR (solid film, $\left.\mathrm{cm}^{-1}\right) ; \quad \nu_{\mathrm{CH}} 3050,2920$, and 2850; $\nu_{\mathrm{C}=\mathrm{O}} 1720$; $\delta_{\mathrm{CH}} 1430$ and $1360 ; \nu_{\mathrm{C}-\mathrm{O}-\mathrm{C}} 1240 ; \nu_{\mathrm{O}-\mathrm{C}-\mathrm{O}} 1120$ and $1020 ; \delta_{\mathrm{C}=0} 940 ; \delta_{\mathrm{CH}} 850$ and 720 (pyrene ring). NMR (hexadeuterio acetone): $\tau 8.65$ (multiplet, $\mathrm{CH}_{2}$ in acetal ring); $\tau 8.18$ (multiplet, $\mathrm{CH}_{2}$ ); $\tau 7.95$ (singlet, $-\mathrm{O}-\mathrm{CO}-\mathrm{CH}_{3}$ ); $\tau 6.30$ (multiplet, $\mathrm{O}>\mathrm{CH}$-pyrene); $\tau 5.10$ (multiplet, $-\mathrm{CH}-\mathrm{O}-$ $\mathrm{CO}-$ ); $\tau 1.80$ (multiplet, pyrene ring protons).

\section{Electrical Measurements}

Measurements of the permittivities and the

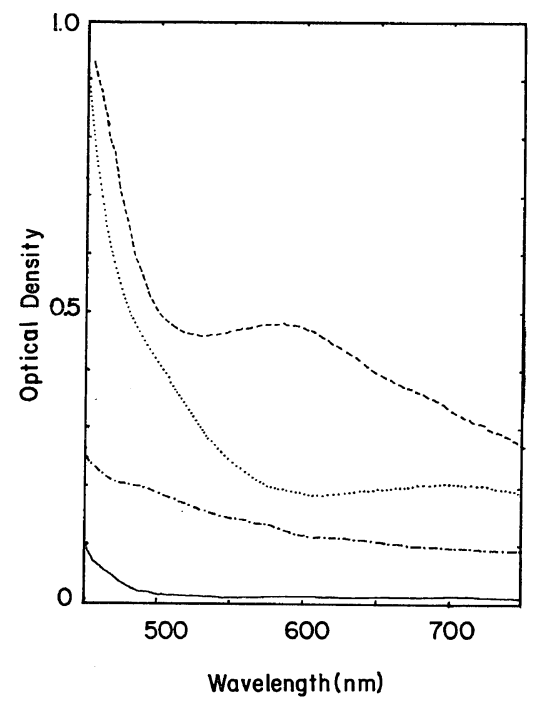

Figure 1. Absorption spectra of the acetylated acetals (I) and (II) and their charge-transfer complexes with TCNQ in solid films: _- the acetylated acetal $(\mathbf{I}) ;-\cdots$, the acetylated acetal $(\mathbf{I})-$ TCNQ; -.-.-, the acetylated acetal (II); ....., the acetylated acetal (II)-TCNQ. 
dielectric losses were carried out with a G. R. Capacitance Bridge Type 1615A at room temperature. The films of the acetylated acetals were prepared from methylene chloride solutions by means of the solvent cast method, and the films of the charge-transfer complexes with TCNQ were likewise prepared from methylene chloride solutions containing 5-wt\% TCNQ with respect to the acetylated acetals. The absorption spectra of the charge-transfer complexes of the acetylated acetals with TCNQ show charge-transfer bands with maxima at around $710 \mathrm{~nm}$ for the acetylated acetal (II) and at around $580 \mathrm{~nm}$ for the acetylated acetal (I) in the film state. They are shown in Figure 1.

\section{RESULTS AND DISCUSSION}

Syntheses of the 9-Acridinealdehyde Acetal (I) and the 3-Pyrenealdehyde Acetal (II) of Poly(vinyl alcohol)

The results of the acetalization of poly(vinyl alcohol) with 9-acridinealdehyde or 3-pyrenealdehyde carried out under various reaction conditions are summarized in Table I. The characterizations of the acetals formed are described in the experimental section. When water was used as a solvent, the reaction system rapidly became heterogeneous, resulting in very low degrees of acetalization, as shown in the reaction of 9-acridinealdehyde. When the reaction was carried out in a dioxane-water $(60: 40)$ binary solvent at $80^{\circ} \mathrm{C}$, the reaction system was homogeneous throughout the reaction; at $60^{\circ} \mathrm{C}$, however, it became heterogeneous during the reaction. Under homogeneous reaction conditions the degree of acetalization of the poly(vinyl alcohol) with the 9-acridinealdehyde reached $32.6 \%$. Reacetalization of the acetal could increase the degree only slightly, to $34.2 \%$. In the case of 3-pyrenealdehyde the degree of acetalization did not exceed $54.15 \%$ under any reaction conditions examined. The relatively low degree of acetalization of poly(vinyl alcohol) has also been found with 9anthraldehyde, i.e., $43.8 \% .^{2}$ These values are much lower than the theoretical value of the maximum degree of acetalization, $86.46 \%$, reported by Flory $^{3}$ for the formalization of poly(vinyl alcohol). It is thought that the steric hindrance caused by the bulky polyacene ring is responsible for the relatively low degree of acetalization. Therefore, we carried out a calculation of the maximum degree of acetalization with the polyacenealdehyde by modifying the method reported by Flory, ${ }^{3}$ namely, by taking account of the steric hindrance caused by the bulky polyacene ring.

Calculations of the Maximum Degree of Acetalization by Taking Account of Steric Hindrance Caused by Bulky Polyacene Rings

The following four cases should be considered when one takes account of the steric hindrance caused by the bulky polyacene ring: case (1), one aldehyde occupies three hydroxyl groups; case (2), one aldehyde occupies four hydroxyl groups; case (3), one aldehyde occupies five hydroxyl groups; and case (4), one aldehyde occupies six hydroxyl groups.

The average number of the unreacted hydroxyl groups per molecule at the end of the reaction will be termed $S_{n}$. The subscript $n$ means that the polymer originally contained $n$ hydroxyl groups in the chain.

Case (1): Obviously, $S_{0}=0, S_{1}=1, S_{2}=2$, and $S_{3}=1$. When $n=4$, the first reaction may occur in any one of the following three ways:

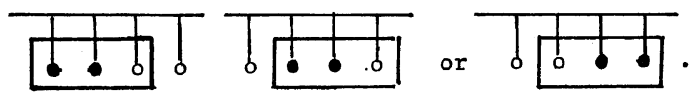

where $\bigcirc$ and - represent the unreacted and reacted hydroxyl groups, respectively, and the three hydroxyl groups covered by the polyacene ring are surrounded with a solid line. Hence $S_{4}=\left(2 S_{1}+S_{1}+3\right) / 3$. When $n=5$, the first reaction may occur in any one of the following four ways:

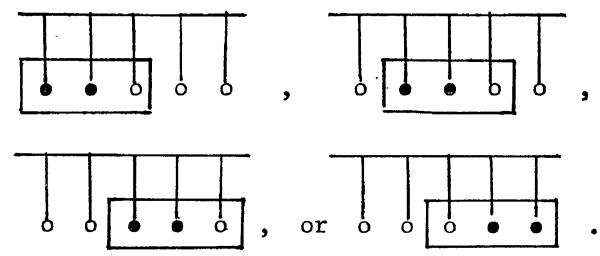

Hence $S_{5}=\left(2 S_{1}+2 S_{2}+S_{2}+4\right) / 4$.

Continuing in this manner, one can obtain the general equation

$$
\begin{aligned}
S_{n}= & {\left[2 \left(S_{1}+S_{2}+S_{3}+\cdots\right.\right.} \\
& \left.\left.+S_{n-3}\right)+S_{n-3}+(n-1)\right] /(n-1) .
\end{aligned}
$$


Table I. Acetalization of poly(vinyl alcohol) with 9-acridinealdehyde or 3-pyrenealdehyde

\begin{tabular}{|c|c|c|c|c|c|c|c|c|c|c|c|c|}
\hline \multirow{2}{*}{ Exptl no. } & \multirow{2}{*}{ PVA, mol/l } & \multirow{2}{*}{\multicolumn{2}{|c|}{ Aldehyde $(\mathrm{mol} / \mathrm{l})$}} & \multirow{2}{*}{\multicolumn{2}{|c|}{ Acid $(\mathrm{mol} / \mathrm{l})$}} & \multirow{2}{*}{ Solvent } & \multirow{2}{*}{ Temp, ${ }^{\circ} \mathrm{C}$} & \multirow{2}{*}{ Time, hr } & \multicolumn{3}{|c|}{ Elementary analyses } & \multirow{2}{*}{$\begin{array}{c}\text { Acetalization } \\
\text { degree }\end{array}$} \\
\hline & & & & & & & & & $\mathrm{N}, \%$ & C, $\%$ & $\mathrm{H}, \%$ & \\
\hline 1 & 0.10 & 9-Aca & 0.10 & $\mathrm{HCl}$ & 0.80 & Water & 60 & 48 & 0.31 & - & - & 3.9 \\
\hline 3 & 0.20 & 9-Ac & 0.30 & $\mathrm{HCl}$ & 1.60 & $\begin{array}{c}\text { Dioxane-Water } \\
(60: 40)\end{array}$ & 60 & 48 & 0.40 & - & - & 5.0 \\
\hline 4 & 0.20 & 9-Ac & 0.30 & $\mathrm{H}_{2} \mathrm{SO}_{4}$ & 1.60 & $\begin{array}{c}\text { Dioxane-Water } \\
(60: 40)\end{array}$ & 60 & 48 & 0.61 & - & - & 7.7 \\
\hline $6^{c}$ & & 9-Ac & 0.30 & $\mathrm{HCl}$ & 2.40 & $\begin{array}{c}\text { Dioxane-Water } \\
(60: 40)\end{array}$ & 80 & 48 & 2.55 & - & - & 34.2 \\
\hline 7 & 0.25 & 3-Py & 0.13 & $\mathrm{HCl}$ & 2.00 & $\begin{array}{c}\text { Dioxane-Water } \\
(60: 40)\end{array}$ & 70 & 4 & - & 62.53 & 8.22 & 9.8 \\
\hline 8 & 0.50 & 3-Py & 0.50 & $\mathrm{HCl}$ & 2.00 & $\begin{array}{c}\text { Dioxane-Water } \\
(60: 40)\end{array}$ & 70 & 24 & - & 68.23 & 7.34 & 20.4 \\
\hline 9 & 0.35 & 3-Py & 0.13 & $\mathrm{HCl}$ & 2.40 & $\begin{array}{c}\text { Dioxane-Water } \\
(80: 20)\end{array}$ & 70 & 24 & - & 72.74 & 6.41 & 32.15 \\
\hline
\end{tabular}

a 9-Acridinealdehyde.

b 3-Pyrenealdehyde.

c Reacetalization of the acetal obtained from exptl no. 5 .

d Reacetalization of the acetal obtained from exptl no. 9.

o $p$-Toluene sulfonic acid. 
Likewise, in the other cases, one can obtain the general equation of $S_{n}$ as followsCase (2):

$$
\begin{aligned}
S_{n}= & {\left[2 \left(S_{1}+S_{2}+S_{3}+\cdots\right.\right.} \\
& \left.\left.+S_{n-4}\right)+2(n-3)\right] /(n-3)
\end{aligned}
$$

Case (3):

$$
\begin{aligned}
S_{n}= & {\left[2 \left(S_{1}+S_{2}+S_{3}+\cdots\right.\right.} \\
& \left.\left.+S_{n-5}\right)+S_{n-5}+3(n-3)\right] /(n-3)
\end{aligned}
$$

Case (4):

$$
\begin{aligned}
S_{n}= & {\left[2 \left(S_{1}+S_{2}+S_{3}+\cdots\right.\right.} \\
& \left.\left.+S_{n-6}\right)+4(n-5)\right] /(n-5)
\end{aligned}
$$

Calculations of $S_{n}$ and $S_{n} / n$ for all cases were carried out with the NEAC-2200 Model 700 electron digital computer at this university; the results $v s . n$ and, for comparison, the case reported by Flory without steric hindrance, are shown in Figure 2. It is shown in Figure 2 that every one of the series of $S_{n} / n$ converges very rapidly beyond $n=20$; when $n=500$, the values become 0.1359 for Flory's case, 0.4440 for case (1), 0.5988 for case (2), 0.6805 for case (3), and 0.7393 for case (4).

The degree of acetalization (A.D.) will be defined as

$$
\text { A.D. }(\%)=100\left(1-S_{n} / n\right) \text {. }
$$

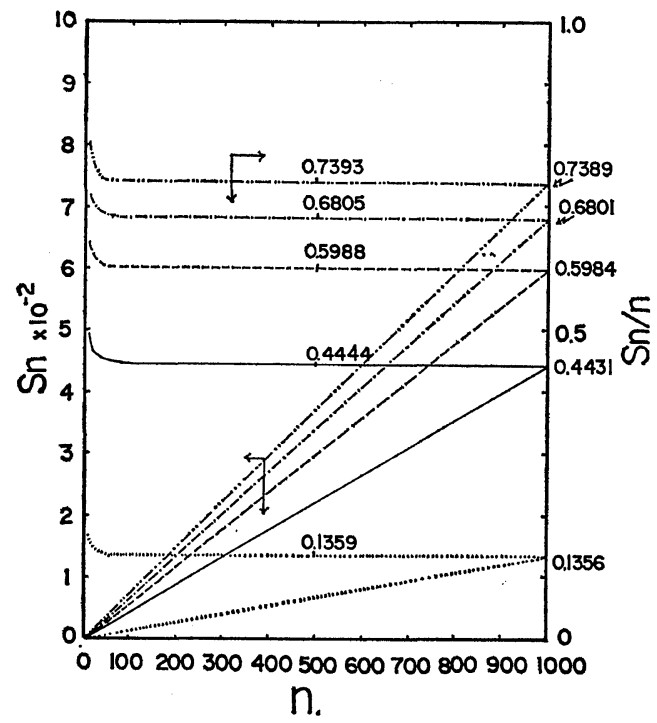

Figure 2. $S n, S n / n$ vs. $n$ plots: - ----, case (2); -.-.-, case (3); $-\cdots \cdot \cdot-$, case (4); and $\ldots . . .$. , Flory's case.
Thus, the maximum degree of acetalization calculated by taking account of the steric hindrance caused by bulky polyacene rings is found to be $55.60 \%$ for case (1), $40.12 \%$ for case (2), $31.95 \%$ for case (3), $26.07 \%$ for case (4), and $86.44 \%$ for Flory's case, respectively. The acetalization with 9-anthraldehyde or 9-acridinealdehyde can be regarded as case (2), and that with 3-pyrenealdehyde as case (1), from their molecular models. The experimental values for the degree of acetalization obtained in the present study are in fair agreement with the calculated ones, although for 9-acridinealdehyde the value obtained experimentally is somewhat lower than that calculated. The reason is not clear at present, but the formation of the salt of 9-acridinealdehyde with hydrochloric acid is perhaps responsible for the lower values of A.D. as compared with that found with 9-anthraldehyde.

\section{Dielectric Properties}

The results of the permittivities measured at $1 \mathrm{kHz}$ are summarized in Table II. The frequency dependences of the permittivities and dielectric losses measured at room temperature are shown in Figure 3. The permittivities of the films of the acetylated acetals (I) and (II) were found to be almost equal (see samples 1, 3 , and 5 in Table II). The permittivities of each of the acetylated acetals increased when complexed with TCNQ, the extent of the increase being dependent on the degree of acetalization (see samples 2, 4, and 6). It is suggested that the charge-transfer interaction between the acridine or pyrene moiety in the acetylated acetal and TCNQ is responsible for the increase

Table II. Dielectric constants and dielectric losses measured at $1 \mathrm{kHz}$ at room temperature

\begin{tabular}{cclccc}
\hline $\begin{array}{c}\text { Sample } \\
\text { No. }\end{array}$ & $\begin{array}{c}\text { Acetal- } \\
\text { ization } \\
\text { degree }\end{array}$ & $\begin{array}{c}\text { TCNQ, } \\
\text { wt\% }\end{array}$ & $\begin{array}{c}\text { Di- } \\
\text { electric } \\
\text { constant }\end{array}$ & $\begin{array}{c}\tan \delta, \\
\%\end{array}$ & $\begin{array}{c}\mathrm{CT} \text { band } \\
\left(\lambda_{\mathrm{CT}}^{\mathrm{max}}, \mathrm{nm}\right)\end{array}$ \\
\hline $1^{\mathrm{a}}$ & 5.0 & 0 & 2.6 & 0.78 & - \\
$2^{\mathrm{a}}$ & 5.0 & 0.15 & 3.1 & 0.81 & 580 \\
$3^{\mathrm{a}}$ & 32.6 & 0 & 2.7 & 0.58 & - \\
$4^{\mathrm{a}}$ & 32.6 & 5.43 & 4.4 & 0.73 & 580 \\
$5^{\mathrm{b}}$ & 20.4 & 0 & 2.6 & 0.58 & - \\
$6^{\mathrm{b}}$ & 20.4 & 4.99 & 2.9 & 0.63 & 710 \\
\hline
\end{tabular}

a Acetylated 9-arcridinealdehyde acetal of PVA.

b Acetylated 3-pyrenealdehyde acetal of PVA. 
Y. Oshiro, M. Yokoyama, Y. Shirota, and H. Mikawa

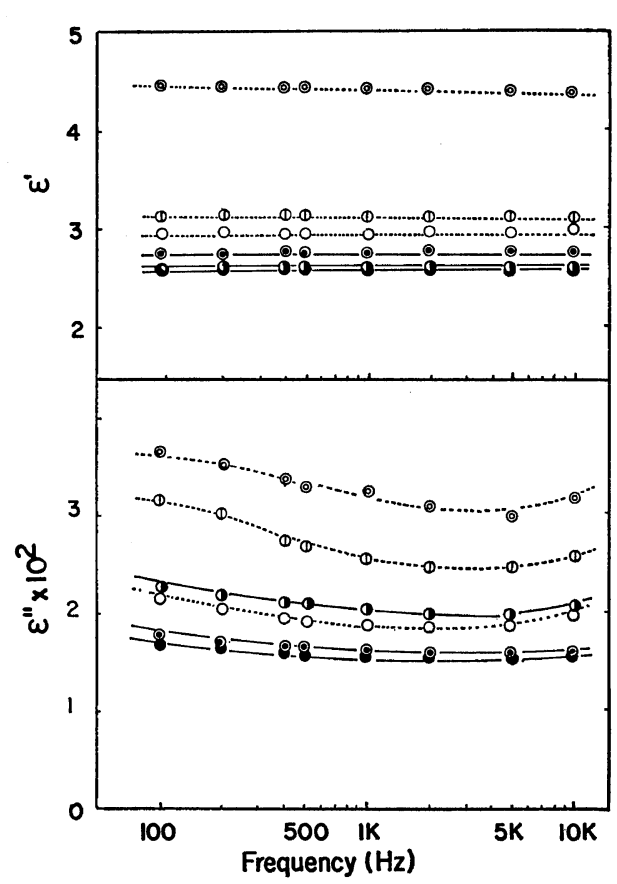

Figure 3. Frequency dependences of permittivities and dielectric losses of the acetylated acetals (I) and (II) and their charge-transfer complexes: sample (1); $\bigcirc$, sample (2); $\odot$, sample (3); (), sample (4); (1), sample (5); (1), sample (6). in the permittivities. Further studies are needed in order to clarify the mechanism of the increase in the permittivities resulting from the charge-transfer complexation.

Acknowledgment. The authors wish to thank Mr. T. Shiba of Nippon Electric Company for measurements of dielectric properties.

\section{REFERENCES}

1. T. Sawamura and S. Saito, Reprint, SPSJ 20th Annual Meeting, Tokyo, Japan, May, 1971, 2, p 405.

2. Y. Oshiro, Y. Shirota, and H. Mikawa, Polymer J., 4, 581 (1973).

3. P. J. Flory, J. Amer. Chem. Soc., 72, 5052 (1950).

4. A. Kaufmann and L. G. Vallette, Ber., 45, 1739 (1912).

5. K. Tanikawa, T. Ishizuka, K. Suzuki, S. Kusabayashi, and H. Mikawa, Bull. Chem. Soc. Japan, 41, 2719 (1968).

6. W. O. Herrman, Ber., 60, 1658 (1927). 